\title{
TRABAJADORES MIGRANTES Y ACTIVISMO SINDICAL EN LOS MÁRGENES: LOS CENTROS DE TRABAJADORES COMO CAMPO ORGANIZATIVO
}

\section{MIGRANT WORKERS AND LABOR ACTIVISM IN THE MARGINS: WORKER CENTERS AS AN ORGANIZATIONAL FIELD}

Beltrán Roca* y Emma Martín-Díaz ${ }^{* *}$

Resumen: Los centros de trabajadores son organizaciones comunitarias que prestan servicios, intervienen politicamente a y movilizan a trabajadores de bajos salarios, principalmente migrantes, en Estados Unidos. El presente artículo analiza los centros de trabajadores como campo organizativo, prestando atención a los factores que originan tanto isomorfismo como heterogeneidad. A partir del estudio etnográfico llevado a cabo en el área metropolitana de Nueva York, se concluye que ante un contexto de segmentación del mercado de trabajo y de ausencia de implicación sindical en los mercados de trabajo secundarios, la respuesta de los trabajadores migrantes se ha concentrado en el desarrollo de formas organizativas propias que presentan como principal activo el recurso a la redes que se conforman y (re) crean para adaptarse. Asimismo, como campo organizativo, las redes entre estas organizaciones implican tanto colaboración como rivalidad. En este sentido, se identifican dos grandes modelos: uno que tiende a la institucionalización y otro que ponen énfasis en la acción directa en los lugares de trabajo.

Palabras clave: centros de trabajadores; sindicalismo comunitario; sindicatos; campo organizativo; trabajadores migrantes.

* Universidad de Cádiz.

** Universidad de Sevilla. 
Abstract: Worker centers are community-based organizations that provide services, undertake political advocacy and organize low-waged workers, mainly migrants, in the USA. This article attempts to analyze worker centers as an organizational field, paying attention to the factors that foster both isomorphism and heterogeneity. Drawing on an ethnographic study in NYC metropolitan area, it concludes that before a context of segmentation of the labor market and lack of trade union engagement in secondary labor markets, the response of migrant workers has focused on developing their own organizational forms, whose main asset are the networks that are (re) created for adapting. In addition, as an organizational field, the networks among these organizations imply both collaboration and competition. In this sense, the article identifies two main models: one that tends to institutionalization and another that put emphasis on direct action and the workplace.

Key words: worker centers; community unionism; trade unions; organizational field; migrant workers

\section{INTRODUCCIÓN}

El primero de mayo de 2019, la ciudad de Nueva York y su área metropolitana estaban repletos de acciones de protesta. A diferencia de Europa, en el que esta fecha moviliza a las organizaciones sindicales, en Estados Unidos son las organizaciones de migrantes las que adquieren protagonismo. Por la mañana, una coalición liderada por el centro de trabajadores Make the Road New York y el local 32BJ del sindicato de servicios SEUI (Service Employees International Union) reivindicaba permisos de conducir para migrantes en situación irregular en la costa de New Jersey, frente a la Estatua de la Libertad y la isla Ellis, que durante la primera mitad del siglo XX sirvió de edificio de aduana desde el que se recibía a la población migrante procedente de Europa. Otras coaliciones en defensa de las personas migrantes, que incluían a otros sindicatos, centros de trabajadores y organizaciones de migrantes, organizaban una marcha que partía de la rotonda de Colón, en la esquina sudeste de Central Park. Otros grupos protestaban ante uno de los edificios Trump por las leyes anti-inmigrantes del gobierno federal. El Laundry Workers Center, un centro de trabajadores que organiza a trabajadores mi- 
grantes en las lavanderías, organizaba un piquete frente a una lavandería en East Harlem, a la que acudían unas 70 personas de diversas organizaciones aliadas. Brandworkers, otro centro de trabajadores cuya sede está en Queens, celebraba un acto en un bar de Manhattan para recaudar fondos para sus próximas campañas de organizing ${ }^{1}$ en la industria alimentaria.

A caballo entre la lucha sindical y la organización comunitaria, los centros de trabajadores ocupan un lugar destacado en la ciudad de Nueva York, organizando a trabajadores precarios, en la mayoría de las ocasiones migrantes (Milkman, 2014). Los centros de trabajadores son organizaciones comunitarias que dan apoyo a trabajadores de bajos salarios (Fine, 2006). A través de la prestación de servicios, la presión política y el organizing tratan de mejorar las condiciones laborales y vitales de este sector de la clase obrera que carece de otras herramientas para defender sus derechos. Una de las características de estas organizaciones es que entienden que la movilización laboral y comunitaria deben ser llevadas a cabo de manera simultánea. Los problemas de vivienda, de inmigración, opresión racial o derechos sociales, son abordados de manera conjunta a la de la opresión en el trabajo. Esto los convierte en claros ejemplos de lo que se conoce como "sindicalismo comunitario".

El presente artículo se plantea, primero, analizar las causas por que los centros de trabajadores se han constituido en Estados Unidos como un campo organizativo diferenciado, en determinadas áreas de las relaciones laborales en las que los sindicatos no intervienen o tienen dificultades para intervenir; y, segundo, identificar si existe tendencia hacia el isomorfismo o hacia la heterogeneidad entre estas organizaciones, y qué factores están detrás de dichas tendencias $^{2}$. Nueva York es una de las ciudades estadounidenses que

1 El término anglosajón organizing tiene una difícil equivalencia al español, por eso se ha preferido dejarla en inglés. Se refiere a una estrategia sindical que trata de realizar campañas intensivas de afiliación, organización y movilización sindical, en ciertas ocasiones acercándose a los trabajadores en sus comunidades y tratando de reclutar sectores tradicionalmente no representados, como migrantes, jóvenes o precarios (Roca, 2016). Una parte de la práctica sindical española se ha definido como "para-organizing" (Martínez Lucio, 2017).

2 Los autores de este artículo quieren agradecer el apoyo de la profesora Ruth Milkman durante el trabajo de campo en Nueva York. No obstante, la responsabilidad del contenido del artículo recae exclusivamente en los autores. La investigación se enmarca en dos proyectos de investigación financiador por el 
concentra un mayor número de centros de trabajadores y en la que éstos llevan a cabo una actividad más dinámica (Fine, Narro y Barnes, 2019), por lo que constituye un entorno privilegiado para el estudio de este tipo de entidades.

El artículo se estructura de la siguiente manera: en primer lugar, se presenta la perspectiva teórica empleada, que combina la teoría de segmentación del mercado de trabajo con una versión actualizada del concepto neoinstitucionalista de campo organizativo. A continuación, se describe la metodología empleada, que ha sido principalmente cualitativa, y se hace referencia a la fiabilidad del estudio más amplio en el que se enmarca el artículo. Posteriormente, se ofrece una visión general del contexto de las migraciones transnacionales en Nueva York y su impacto sobre los mercados de trabajo. Luego se ofrece una descripción general de los centros de trabajadores en el área metropolitana y se detiene, a continuación, en la conformación de un campo organizativo diferenciado, prestando atención no sólo a las relaciones cooperativas entre centros de trabajadores, sino también a relaciones conflictuales. Finalmente, se sintetizan las principales conclusiones e implicaciones del estudio.

\section{PERSPECTIVA TEÓRICA: SEGMENTACIÓN DEL MERCADO DE TRABAJO, AGENCIA Y CAMPO ORGANIZATIVO}

\subsection{Mercado de trabajo, sindicalismo y centros de trabajadores}

La inserción laboral de los inmigrantes en los mercados de trabajo de las sociedades de destino ha sido analizada mayoritariamente a partir de los años sesenta desde la óptica segmentarista de los mercados de trabajo. Esta aproximación parte de diferentes enfoques que tienen en común el cuestionamiento de la teoría neoclásica de los mercados de trabajo perfectos. En los años sesenta una serie de trabajos empíricos (Gordon, 1972) ponen de manifiesto que los sec-

Ministerio de Ciencia, Innovación y Universidades: "Redes de solidaridad de trabajadores migrantes en Estados Unidos y Europa” (ref. CAS18/00018), del programa José Castillejo, y "Nuevas 'movilidades' intraeuropeas: decisiones sobre el trabajo, la familia y la política de los españoles 'transmigrantes"' (CSO201784618-P), del Plan Estatal de Investigación Científica y Técnica y de Innovación. 
tores que ocupan las posiciones más bajas de la escala laboral y salarial parecen funcionar con patrones diferentes al resto. Aparece así dentro del enfoque institucionalista la teoría dualista de los mercados de trabajo (Piore, 1969), que es reformulada desde la economía radical, cambiando el enfoque del análisis de los mercados de trabajo al análisis de los procesos de trabajo (Bowles y Gintis, 1975; Gordon, Edwards y Reich, 1986). Sin embargo, algunos autores, como Mottura (1992), Pugliese (1992), King y Black (1997), o Martín Díaz, Melis Maynar y Sanz Casas (2004), cuestionan la validez del modelo en el contexto posfordista, llegando a la conclusión de que las dinámicas de la globalización económica y la creciente financiarización de la economía imponen nuevas formas de funcionamiento en los mercados de trabajo que implican nuevos modelos migratorios. Aparece el concepto de cadenas globales de valor que tiene su origen en la teoría del sistema mundo y, en concreto, en la noción de las cadenas de mercancías (Hopkins y Wallerstein, 1977), sin embargo, lo que pretende enfatizar este planteamiento no es la internacionalización de la producción, sino su globalización (Sassen, 2014). Para Sassen, además de los efectos de la globalización en el incremento de las desigualdades y en la precarización de amplias capas de trabajadores, lo que tiene lugar es la aparición de nuevos modelos de movilidad geográfica, particularmente entre la población inmigrante, que es expulsada masivamente de sus países de origen. En este sentido, lo que tiene lugar con la globalización es una aceleración de las transferencias transnacionales de capital y de trabajo.

Estas transferencias pueden verse des dos formas contradictorias entre sí. Algunos lobbies económicos, como La New American Economy (NAE) subrayan que los trabajadores inmigrantes son las personas que mejor se adaptan a las nuevas condiciones de trabajo en las economías globalizadas por su condición de gap fillers. Así, para autores como Fielding (2010), la precarización de los empleos, lejos de extremar las condiciones de vulnerabilidad de los trabajadores migrantes conforme a las predicciones de las teorías segmentaristas, crean condiciones favorables para éstos, particularmente en tiempos de recesión debido precisamente a esta condición. Existen, pues, dos posiciones: la segmentarista, que enfatiza los aspectos estructurales entre los que destaca la vulnerabilidad que se produce cuando se introducen en los mercados de trabajo categorías ajenas a la profesionalidad, como el género, la edad, o la condición de ciudadanía/extranjería, y la resiliente, que destaca la capacidad de 
agencia de estos trabajadores precisamente por sus condiciones de flexibilidad y adaptación a los nichos laborales precarizados que les otorga su condición de extranjeros.

Nuestro enfoque intenta mostrar, a partir del estudio de los centros de trabajadores como campo organizativo diferenciado, cómo esta posición más favorable de los migrantes para enfrentar los periodos de crisis y precarización no proviene exclusivamente de su situación de trabajadores paradigmáticamente flexibles, sino particularmente de su capacidad de resiliencia a la vulnerabilidad, resistencia generada a partir de los modelos específicos de inserción laboral, sus estrategias organizativas para enfrentar estas condiciones de inserción, y las experiencias previas de estos trabajadores en sus sociedades de origen.

Pero la resiliencia y agencia de los trabajadores migrantes no puede entenderse sin prestar especial atención al papel de las organizaciones sindicales en los mercados de trabajo y sociedades de destino. A partir del análisis de experiencias en Europa y Estados Unidos, Pennix y Roosblad (2001) apuntan los retos que suponen para los sindicatos la restructuración del empleo y la segmentación del mercado de trabajo en base al género o la nacionalidad. Según estos autores, el nuevo contexto trae dos grandes dilemas. El primero es si cooperar o no con las políticas migratorias, políticas que en muchos casos implican la exclusión de derechos ciudadanos a las personas migrantes. El segundo dilema se deriva de dichas políticas, y es aceptar la presencia de inmigrantes, en particular aquellos en situación administrativa irregular. La variedad de respuestas existentes, desde integrar a los migrantes hasta ignorarlos, es tan amplia como contradictoria en muchos casos. Estos dilemas y contradicciones se expresan asimismo en las mismas políticas sindicales, en especial en sus programas de integración. En un trabajo más reciente, Marino, Pennix y Roosblad (2015) revisan estos dilemas argumentando que resolverlos implican que "los sindicatos deben actuar hoy como un actor de la sociedad civil a favor de los inmigrantes en lugar que como grupos de interés relacionados estrictamente con el empleo" (p. 10).

A pesar de las recomendaciones de académicos y expertos, la respuesta real de las organizaciones sindicales a la presencia de migrantes es muy diversa: desde utilizar un discurso abiertamente racista y apoyar políticas anti-inmigración, hasta incorporar clausulas antirracistas en la negociación colectiva, formar a los afiliados 
y cuadros en cuestiones de igualdad, y participar en coaliciones a favor de los derechos de las personas migrantes. Connolly, Marino y Martínez Lucio (2019) proponen una perspectiva multidimensional para comprender la respuesta de las organizaciones sindicales a la inmigración y la inclusión social. Esta perspectiva debe tener en cuenta: el contexto social, el contexto institucional formal, el contexto político, y el contexto regulatorio y de política social.

Numerosos estudios apuntan a un creciente interés de las organizaciones sindicales por los trabajadores migrantes, un giro hacia su aceptación y defensa (López, 2004). Lo cierto es que, en determinados contextos, los trabajadores migrantes han sido objeto de campañas de organizing e iniciativas de renovación sindical (Murray, 2017). En el caso de Estados Unidos, la experiencia de movilización de las limpiadoras en Los Ángeles a finales de la década de 1990 y, posteriormente, en otras ciudades e industrias, sentó las bases para una relación más estrecha entre los sindicatos y los trabajadores migrantes (Milkman, 2000). Se produjo un giro estratégico en muchos de los grandes sindicatos. No obstante, la inclusión de los migrantes ha sido irregular y, por lo general, limitada (Ness, 2005). Por ejemplo, en numerosas industrias en las que predomina pequeña empresa, trabajadores en situación irregular y los bajos salarios, los sindicatos no han hecho esfuerzos por organizar a los trabajadores, pues esto requiere una enorme inversión de recursos que posteriormente no se van a rentabilizar en forma de cuotas sindicales ${ }^{3}$.

Por el lado del trabajador migrante, el análisis del activismo sindical debe partir de las propias experiencias y subjetividades. En este sentido, Milkman (2000) ha subrayado tres factores que favorecen la movilización de trabajadores migrantes. Primero, su situación de estigmatización que, según la autora, fomenta la solidaridad: "la experiencia de estar en un ambiente de hostilidad, lejos de generar pasividad y miedo, como muchos autores han sugerido, puede en

3 En el sistema estadounidense, el modelo clásico de acción sindical implica realizar en el centro de trabajo/empresa unas elecciones para que la plantilla sea representada por un sindicato. Si se supera esa elección se inicia un periodo de negociaciones para alcanzar un convenio colectivo. Una vez firmado el acuerdo, todos los trabajadores están obligados a pagar la cuota sindical por el hecho de verse acogidos al convenio. Aunque sentencias recientes del Tribunal supremo han anulado la obligatoriedad de abonar la cuota sindical en los empleados públicos, a través de legislaciones de ámbito estatal y acción sindical, los sindicatos están tratando de afrontar dicha amenaza (Semuels, 2018). 
cambio promover solidaridad y organización (así como otras formas de actividad política de oposición)" (Milkman, 2000). Segundo, los antecedentes políticos y sindicales de muchos migrantes. Muchos activistas migrantes de Estados Unidos tienen una trayectoria militante significativa en sus países de origen. En algunos casos, la emigración ha sido una forma de escapar de opresión política y represión violenta en dichos países. Tercero, el papel de las redes de los migrantes. Los investigadores sobre migraciones suelen reconocer la relevancia de las redes sociales y personales en los procesos migratorios. Las redes dan forma a sistemas migratorios, proyectos, comunidades y procesos de integración. En un contexto de falta de recursos económicos y culturales, las redes de los migrantes son un activo fundamental para reconstruir sus vidas y proveer bienestar. Dichas redes también pueden ser un recurso valioso para la acción colectiva.

La ambigüedad de las políticas sindicales hacia los trabajadores migrantes, unida a las circunstancias de opresión en que viven y su inclinación hacia la acción colectiva, forman el caldo de cultivo para la aparición de formas de auto-organización, o al menos, la creación de redes y organizaciones propias que den respuesta a sus necesidades vitales y aspiraciones. En el caso de Estados Unidos, la proliferación de los centros de trabajadores ha hecho más visible estas experiencias, llevando a los estudiosos a hablar de activismo extra-sindical (Sullivan, 2010) o alt-labor (sindicalismo alternativo) (Milkman, 2013). En otras latitudes, la capacidad para reconocer las formas de auto-organización de los trabajadores migrantes y/o precarios ha sido más limitada. En el caso de Reino Unido, por ejemplo, los sindicatos han tenido mayor dificultad para reconocer las propias organizaciones de los migrantes y conectar con sus redes (Connolly, Marino y Martinez Lucio, 2014). A pesar de ello, investigadores de las relaciones laborales han apuntado la necesidad de incluir las demandas de los trabajadores migrantes en la identidad, estructura y canales de comunicación de los sindicatos (Marino et al., 2015).

Los centros de trabajadores constituyen casos de sindicalismo comunitario, en el sentido de que implican un cambio sustancial en las prácticas espaciales sindicales: principalmente, no limitarse al lugar de trabajo, sino ampliara los ámbitos geográficos en los que los trabajadores desarrollan su vida familiar y colectiva (McBride y Greenwood, 2009). Como Wills y Simms (2004) han puesto de manifiesto, no es exactamente preciso afirmar que esta espacialidad sea 
totalmente novedosa, pues las formas originarias de sindicalismo tenían un fuerte componente comunitario. Sólo el modelo sindical posterior a la Segunda Guerra Mundial adoptó determinadas prácticas espaciales y de representación que alejaron al sindicato de la comunidad. La reestructuración neoliberal de la economía a partir de la década de 1970 sentó las bases para el resurgimiento de nuevas formas de sindicalismo comunitario, como los centros de trabajadores.

Las formas de auto-organización laboral de trabajadores precarios y migrantes, que por lo general incluyen reivindicaciones que van más allá de los problemas estrictamente laborales (Milkman, 2013), vienen alimentadas por la creciente precarización y desregulación del empleo y el trabajo, como resultado de la restructuración capitalista y las transformaciones tecnológicas. Sin embargo, independientemente de cómo se conceptualicen estas organizaciones y redes, y de sus alcances y limitaciones, su existencia tiene tres grandes implicaciones para el estudio de las relaciones laborales y la acción colectiva. Primero, que es preciso dejar de pensar en el sindicato como la institución única (o incluso central) dentro del movimiento obrero. Segundo, que estas entidades, al estar menos sujetas a regulaciones estatales e inercias burocráticas internas, suelen ser una fuente de innovación social. Con frecuencia estas innovaciones surgen en los márgenes, pero con el tiempo terminan siendo adoptadas por la mayoría de las organizaciones con las que comparten espacio. Tercero, los sindicatos deben desarrollar un acercamiento más inclusivo hacia estas redes, dado que normalmente han aparecido en aquellos espacios en los que los repertorios de acción de los sindicatos carecen de eficacia debido a la extensión de la subcontratación y la restructuración del empleo (Milkman, 2013). De hecho, es preciso apuntar que el sindicalismo comunitario en Estados Unidos es principalmente entendido como un modelo organizativo, mientras que en Europa se entiende más como un proceso (Black, 2005). La proliferación de los centros de trabajadores en Estados Unidos es precisamente la causa de la diferencia de planteamientos sobre sindicalismo comunitario con Europa, pues en Europa no hay un grupo de organizaciones tan extenso y bien delimitado que esté operando junto a los sindicatos en el ámbito de la opresión en los lugares de trabajo. 


\subsection{Los centros de trabajadores como campo organizativo}

Investigaciones recientes han puesto de manifiesto que los centros de trabajadores constituyen un campo organizativo diferenciado (Fine, Narro y Barnes, 2019). En su formulación original, DiMaggio y Powell (1983) señalaban que un conjunto de organizaciones sometidas a unas mismas presiones normativas (por ejemplo, valores culturales compartidos), coercitivas (especialmente mediante la regulación estatal) y miméticas (por procesos de imitación entre ellas), tienden hacia el isomorfismo, esto es, a presentar unas mismas estructuras organizativas, y a constituir espacios sociales diferenciados, generando sus propias identidades y reglas.

Aunque la tesis de la tendencia al isomorfismo ha sido ampliamente discutida (Hambrick, Finkelstein, Cho y Jackson, 2005), especialmente en los estudios sobre el tercer sector (Leiter, 2005; Roca, 2014, 2019), el concepto de campo organizativo ha servido para estudiar las relaciones - cooperativas y competitivas- entre organizaciones que operan en una misma industria o sector (Roca, 2006). En el ámbito de los centros de trabajadores, investigaciones previas han subrayado tendencias a la burocratización y la profesionalización (Jenkins, 2002).

En el caso de los centros de trabajadores, aunque la noción de campo organizativo y el enfoque neoinstitucionalista ayudan a explicar las dinámicas por las que se han extendido este tipo de organizaciones, es preciso hacer tres consideraciones: primero, aunque haya tendencias isomórficas, los centros de trabajadores presentan diferentes tipos de identidades, estrategias, programas y repertorios de acción; segundo, los estudios sobre centros de trabajadores enfatizan las creación de redes y alianzas, entre distintos centros de trabajadores, pero también con sindicatos y otras organizaciones de la sociedad civil, pero tienden a obviar las relaciones conflictuales entre ellas. Como Heery, Williams y Abbott (2012) han puesto de manifiesto, las relaciones entre sindicatos y organizaciones de la sociedad civil son complejas, y oscilan entre la indiferencia, el conflicto y la cooperación. Efectivamente, todo campo es un espacio de lucha, incluso entre organizaciones que comparten formalmente unas mismas finalidades; tercero, aunque la perspectiva neoinstitucionalista se centra en las estructuras organizativas, el estudio de los centros de trabajadores precisa investigar también los marcos de referencia y los repertorios de protesta, además del contexto de 
economía política de las migraciones transnacionales y los mercados de trabajo.

Las cifras del número de centros de trabajadores en Estados Unidos son unas primeras señales de la aparición y consolidación de un campo organizativo. En 1992 sólo había cuatro centros, en 2003 el número ascendió a 137, y en 2010 se han identificado más de 200 (Fine, 2011). A este respecto, es preciso apuntar que, a pesar del crecimiento en el número de organizaciones, la capacidad movilizadora de los centros de trabajadores no ha evolucionado en la misma dirección. El punto álgido de actividad se sitúa entre finales de la década de 1990 y el principio del 2000, años en los que desarrollaron innovadoras formas de organización y oposición ante la opresión laboral de los trabajadores migrantes (Jarayaman y Ness, 2005). Los cambios en el contexto general y la evolución interna de estas organizaciones, pueden explicar las causas por los que los niveles de experimentalidad, innovación y radicalidad se han ido reduciendo en los últimos años. En todo caso, los centros de trabajadores, como población organizativa dentro de un campo más amplio (el del movimiento obrero), constituyen un escenario privilegiado para conocer los límites y alcances de formas comunitarias de militancia sindical, en concreto aquella en el que el protagonismo reside en los trabajadores migrantes.

\section{METODOLOGÍA Y TRABAJO DE CAMPO}

La investigación sobre los centros de trabajadores en la ciudad de Nueva York, en la que se enmarca este artículo, se desarrolló a través de varias etapas y actividades en la primera mitad del año 2019. En primer lugar, se elaboró un inventario de centros de trabajadores activos en el área metropolitana consultando las páginas web y cuentas de Facebook de dichas organizaciones. Esto ayudó a recopilar documentos sobre estas organizaciones que posteriormente serían analizados. A continuación, se llevaron a cabo 13 entrevistas semi-estructuradas a participantes de una muestra de organizaciones. Los participantes tenían diferentes estatus dentro de la organización, en concreto eran directores, plantilla (principalmente organizers) y miembros (personas inscritas, generalmente con poco control sobre la toma de decisiones). La muestra de or- 
ganizaciones pretendía cubrir la diversidad de modelos de centros de trabajadores en función de relaciones orgánicas con sindicatos, tamaño, industria en la que intervienen y modelos de actividad (según el peso relativo de la presión política, la provisión de servicios y la organización en los lugares de trabajo). Para la realización de entrevistas se utilizó un guion que adaptó al perfil del participante, pero en términos globales prestaba atención a las experiencias y trayectorias del entrevistado, la historia de la organización, la estructura organizativa, el sistema de financiación, las relaciones externas y la política de alianzas, los repertorios de acción y las representaciones del entrevistado acerca del empleo, la situación de los migrantes, el sistema político, las organizaciones sindicales y los centros de trabajadores.

Las entrevistas cualitativas fueron complementadas con observación participante en una veintena de eventos, como reuniones, manifestaciones, piquetes, boicots secundarios, debates comunitarios, etc. En todos los eventos se informó a los responsables y asistentes de la finalidad de la participación del investigador y se obtuvo su consentimiento.

La información producida a partir de estas técnicas fue posteriormente codificada y sometida a un análisis categorial temático (Braun y Clark, 2006). El análisis se centró principalmente en los siguientes ejes: estructura organizativa, financiación, redes, representaciones, motivaciones, actividades y prácticas espaciales.

La utilización de tres técnicas de investigación diferentes —análisis de documentos, entrevista cualitativa y observación participante-permitió triangular la información de manera efectiva (Marying, 2007). La representatividad teórica de la muestra de organizaciones y participantes, el uso exhaustivo de la información recogida y la triangulación de técnicas ha garantizado la fiabilidad de la investigación (Silverman, 2009).

En este artículo se ha empleado información extensiva sobre los centros de trabajadores, sin detenerse en estudios de caso, con el propósito de examinar las dinámicas comunes que han llevado a conformar un campo organizativo diferenciado, identificando las tendencias hacia el isomorfismo, pero también las que explican la diversidad existente. 


\section{CONTEXTO: MIGRACIONES TRANSNACIONALES Y MERCADO DE TRABAJO EN EL ÁREA METROPOLITANA DE NUEVA YORK}

La población inmigrante es un pilar fundamental en la vida económica y social de la ciudad de Nueva York. La administración municipal calcula que en 2016 había en la ciudad un total de 3,1 millones de personas migrantes, que constituían el 38 por ciento de la población y un 45 por ciento de la fuerza laboral (MOIA, 2018). En 1990 los migrantes constituían tan sólo el 31 por ciento de la mano de obra, lo que indica que en los últimos treinta años los flujos migratorios con destino a la ciudad se han intensificado. La consolidación de Nueva York como una ciudad global, que concentra flujos de información y capitales, conlleva una creciente informalización de determinados sectores de actividad económica que son demandadas en ellas (Sassen, 2005). Dichos sectores de actividad informalizados y desregulados constituyen la base de lo que Piore (1979) denominó mercado de trabajo secundario, caracterizado por inestabilidad contractual, bajos salarios, y mayor exposición a riesgos laborales. La existencia de dicho mercado secundario ha sido un atractivo para que un número creciente de trabajadores extranjeros eligieran Nueva York como destino de sus proyectos migratorios.

En términos generales, los trabajadores migrantes, poseedores de un menor poder social de negociación, se han concentrado en estos sectores informalizados; no obstante, es preciso matizar que el poder de negociación de los migrantes ha dependido de su situación administrativa y, por tanto, de la evolución del marco regulatorio de las migraciones. En 2016, aproximadamente el 54 por ciento de las personas migrantes habían adquirido la ciudadanía estadounidense, otro sector estaba a través de la Green Card u otro tipo de estatus administrativo, y alrededor de 560.000 personas se encontraban en situación administrativa irregular ("indocumentados", según la terminología estadounidense). Las políticas anti-inmigración de la administración federal de Trump han hecho retroceder el número de personas en situación irregular, pues en el año 2008 la cifra en Nueva York ascendía a 610.000 personas (MOIA, 2018).

A nivel geográfico, la población migrante se concentra principalmente en determinados barrios de los distritos de Queens y Brooklyn, aunque en determinadas zonas de Manhattan, como West Harlem, y el Bronx, también hay altos niveles de este sector pobla- 
cional. Según país de procedencia, el principal grupo poblacional es de República Dominicana (422 mil personas), seguida de China (318 mil), México (183 mil) y Jamaica (176 mil). Otros países de origen, por orden de importancia son: Guyana, Ecuador, Haiti, Trinidad y Tobago, Bangladesh y India (MOIA, 2018, p. 12).

El 40,9 por ciento de las personas migrantes habla español, y se calcula que un 63 por ciento de las personas en situación irregular no maneja el inglés (MOIA, 2018, p.13). En efecto, la población hispanohablante es muy importante numérica y socialmente en Nueva York, en especial en determinados enclaves étnicos de los distritos de Brooklyn y Queens (como, por ejemplo, Corona o Jackson Heights). El alto número de población de origen latinoamericano y su concentración en determinados enclaves étnicos ha contribuido a la formación de una identidad panhispana o latina, alimentada también por determinados medios de comunicación, cuadros políticos y organizaciones de la sociedad civil (Mora, 2014). Esta identidad panhispana constituirá la base de una buena parte del activismo de los centros de trabajadores de la ciudad de Nueva York.

Pero el activismo de la población migrante no puede comprenderse sin tener en cuenta las experiencias de explotación laboral y opresión. Las estadísticas reflejan la fuerte vulnerabilidad de las personas en situación irregular, que presentan menores niveles de formación, cobertura médica, ingresos y mayores niveles de problemas relacionados con la vivienda (hacinamiento y dificultad de acceso) (MOIA, 2018). En relación a las relaciones laborales, la población inmigrante de Nueva York presenta niveles similares de participación en el empleo a la población nacida en Estados Unidos. Alrededor de un 65 por ciento de los residentes en el municipio de más de 16 años se encuentra trabajando. No obstante, entre la población en situación irregular esta cifra asciende al 77,3 por ciento. La inmigración irregular hacia Nueva York es principalmente laboral (MOIA, 2018, p. 18).

Respecto a los sectores en los que se emplea mano de obra migrante, la tabla 1 contiene los datos precisos. Los principales sectores son educación, salud y servicios humanos (25\%), servicios de entretenimiento $(12 \%)$, profesionales $(10 \%)$ y comercio ( $8 \%)$. Como se puede observar, trabajan en los sectores que mayor empleo demandan en la ciudad según la agencia de estadísticas laborales (BLS, 2019). 
TABLA 1

PRINCIPALES SECTORES EN LOS QUE TRABAJAN PERSONAS MIGRANTES EN LA CIUDAD DE NUEVA YORK

\begin{tabular}{|l|c|}
\hline \multicolumn{1}{|c|}{ Industria } & $\begin{array}{c}\text { \% del total trabajadores migrantes empleados } \\
\text { en dicha industria }\end{array}$ \\
\hline Educación, salud, servicios humanos & $25 \%$ \\
\hline Servicios de entretenimiento & $12 \%$ \\
\hline Profesionales & $10 \%$ \\
\hline Comercio & $10 \%$ \\
\hline Financiero & $8 \%$ \\
\hline Otros servicios & $7 \%$ \\
\hline Construcción/extracción & $7 \%$ \\
\hline Transportes y suministros & $7 \%$ \\
\hline Manufacturas & $5 \%$ \\
\hline Asuntos internacionales & $3 \%$ \\
\hline
\end{tabular}

Fuente: MOIA 2018, p. 18.

Como diversos estudios ponen de manifiesto, la mano de obra migrante en situación irregular se concentra en determinados segmentos del mercado de trabajo con menores salarios, protección sindical, regulación estatal, y, en general en peores condiciones (Ness, 2005; Avedaño y Hiatt, 2012). Esta posición de los trabajadores migrantes dentro de la economía política de la ciudad de Nueva York es precisamente lo que alimenta su conciencia y su activismo, del que los centros de trabajadores son sólo una expresión.

\section{LOS CENTROS DE TRABAJADORES EN EL ÁREA METROPOLITANA DE NUEVA YORK. UNA RADIOGRAFÍA}

El primer centro de trabajadores como tal en la ciudad de Nueva York, y uno de los primeros de todo Estados Unidos, es el Chinesse Staff and Workers Association (Asociación de Empleados y Trabajadores Chinos), constituido en 1979 con el propósito de organizar a los trabajadores de los restaurantes de Chinatown frente a los abu- 
sos patronales. Ante la incapacidad de los sindicatos para organizar y defender a ese sector de trabajadores migrantes, una serie de activistas optó por crear su propia asociación y desarrollar sus propias formas de organización y acción colectiva. Es cierto que otra organización comunitaria más centrada en la vivienda, la Carroll Gardens Association (Asociación de los Jadines Carroll), que desempeña, entre otras actividades algunas funciones propias de centros de trabajadores, como organizar una cooperativa de niñeras, tiene sus orígenes en el activismo de la década de 1960 y fue formalizada como entidad no lucrativa en 1971, pero la creación de una organización como un centro de trabajadores no se da hasta que se crea Chinesse Staff and Workers Association.

Aunque hay centros de trabajadores que remontan sus orígenes en la década de 1970, no es hasta el año 1992 cuando comienzan a surgir de manera más sostenida nuevos centros de trabajadores (ver figura 1). No puede afirmarse que la proliferación de centros de trabajadores coincida con el aumento de la inmigración a Estados Unidos. La inmigración ha aumentado de manera sostenida, aunque con altos y bajos, desde la década de 1960. Sin embargo, a partir de 1970 se produce en el país un fuerte aumento de la desigualdad, que alimenta una narrativa anti-migratoria (Massey y Pren, 2013). La restructuración capitalista iniciada en la década de 1970, que se manifiesta plenamente ya en la década de 1990, es la que propicia, por un lado, la crisis del movimiento sindical (Lopez, 2004) y, por otro, la difusión de industrias y mercados de trabajo secundarios que emplean a trabajadores con bajos ingresos, en los que se concentran los trabajadores migrantes, sobre todo en servicios, construcción y producción y transporte de materiales (Lesser y Bataova, 2017). La incapacidad de los sindicatos estadounidenses y la necesidad de dar una respuesta a los problemas laborales del importante sector de trabajadores de bajos salarios y migrantes, son la causa que explica la proliferación de los centros de trabajadores.

A finales de la década de 1990 y principios de 2000 los centros de trabajadores se encontraban en su momento de mayor actividad, visibilidad y poder movilizador (Ness, 2005), a partir de enconces su influencia ha disminuido, aunque aun conservan una considerable actividad (Manheim, 2017; Fine, Narro y Barnes, 2019). Independientemente de las consideraciones sobre la vitalidad de estas entidades, estudios recientes han puesto de manifiesto que han consttuido lo que DiMaggio y Powell (1983) denominaron un campo organi- 
zativo, es decir, se trata de un conjunto de organizaciones sometia a unas mismas presiones externas y, por tanto, con estructuras organizativas (pero también repertorios y marcos) muy similares, que los diferencian de otro tipo de organizaciones dentro del movimiento obrero o en el conjunto del Tercer Sector.

El inventorio elaborado ha identificado en la actualidad 32 centros de trabajadores en el área metropolitana de Nueva York, sin contar algunas redes de centros de trabajadores que cuentan con sus propios presupuestos y plantilla. Es preciso matizar que a lo largo de los años, muchos centros de trabajadores han desaparecido, por falta de fondos, por ser absorbidos por otros centros de trabajadores mayores, o porque sus promotores han derivado sus esfuerzos sobre otro tipo de proyectos políticos, laborales o personales. Estos centros de trabajadores no han sido contabilizados y no aparecen reflejados en la Figura 1, aunque referencias a algunos de ellos han aparecido en las entrevistas realizadas y documentos analizados.

FIgURA 1

NÚMERO DE CENTROS DE TRABAJADORES Y AÑO DE CREACIÓN EN EL ÁREA METROPOLITANA DE NUEVA YORK

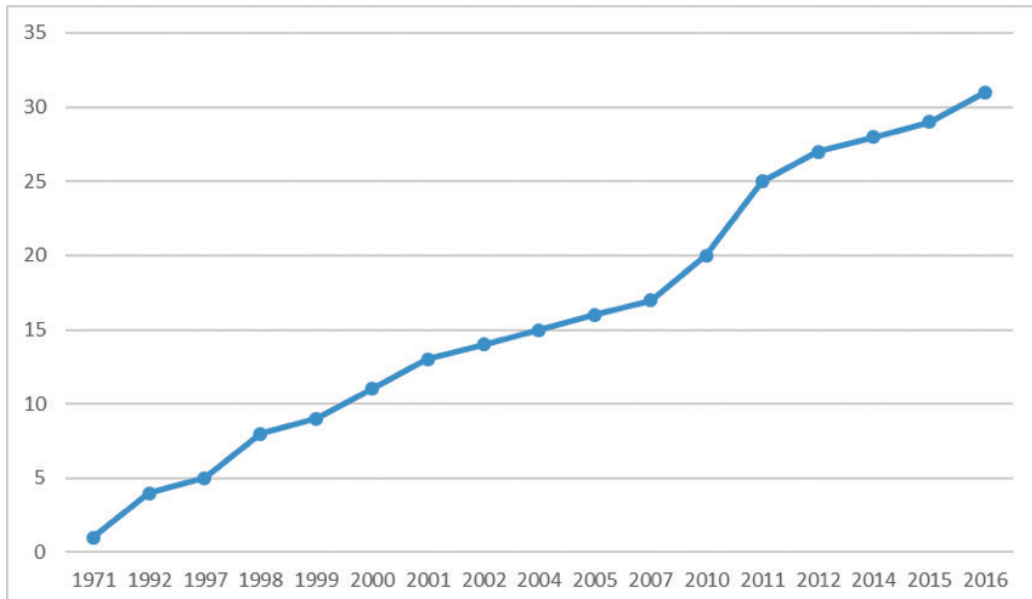

Fuente: elaboración propia. 
Lo que distingue a los centros de trabajadores de otro tipo de actores dentro del movimiento obrero es, entre otras cuestiones, que están formalmente constituidas como entidades no lucrativas (generalmente dentro de la categoría 501(c)3 dentro de la legislación estadounidense), y las actividades que realizan. Mientras los sindicatos tratan de organizar a trabajadores en los centros de trabajo, promoviendo elecciones para que haya representación sindical y, posteriormente, se proceda a negociar un convenio colectivo, los centros de trabajadores tienen otra aproximación a los problemas de justicia laboral. Aunque en algunas ocasiones cooperan con sindicatos establecidos, o constituyen sindicatos independientes, de cara a negociar convenios, los centros de trabajadores suelen trabajar con sectores de trabajadores en las que la sindicalización y la negociación colectiva es si no imposible, al menos extremadamente difícil por la naturaleza del trabajo que se realiza: empleadas domésticas, autónomos, trabajadores subcontratados, vendedores ambulantes, asalariados de pequeñas empresas, conductores de taxi o plataformas electrónicas, etc. En lugar de organizar a esos trabajadores desde los lugares de trabajo, los centros de trabajadores ponen el énfasis en la comunidad. Tratan de acceder a los trabajadores principalmente desde los barrios en los que viven, buscando aliados entre las organizaciones y miembros de esas comunidades, y abordando no sólo problemas estrictamente laborales, sino en muchas ocasiones de diferente índole (vivienda, formación, educación, integración, regularización, etc.). Es por ello por los que los centros de trabajos son entidades de carácter híbrido, que combinan la acción sindical o para-sindical con actividades propias de organizaciones comunitarias.

La tabla 2, elaborada a partir de la consulta a las páginas web y cuentas de Facebook de los centros de trabajadores en la ciudad de Nueva York, refleja las distintas actividades realizan los centros de trabajadores. Como puede observarse, las principales actividades son la presión política, encaminada a influir sobre leyes y políticas públicas que beneficien a los trabajadores migrantes y con bajos salarios, y la educación pública y la formación. Un ejemplo de lo primero, es puede ser la ley local 196, aprobada en 2017, por la que el Ayuntamiento de Nueva York exige a las empresas de construcción que los empleados en el sector tengan acreditadas una serie de horas de formación sobre seguridad y salud en el trabajo. En efecto, dado que es difícil mejorar las condiciones laborales de los trabajadores más vulnerables a través de la negociación colectiva, por haber ele- 
vadas cotas de informalidad, individualización o fragmentación en el empleo, los centros de trabajadores desarrollan una intensa actividad dirigida a promover cambios legislativos a escala municipal o estatal que favorezcan a estos trabajadores (a través de la subida del salario mínimo, el impedimento de la fuga de capital en caso de deudas con los trabajadores, el refuerzo de la seguridad y salud en el empleo, etc.).

La actividad educativa de los centros de trabajadores, por otro lado, suele estar influida en la educación popular latinoamericana tratando de dar formación política y visión crítica, a la vez que se capacita a los trabajadores y se les motiva a participar activamente en la organización. Algunas organizaciones tienen en la oferta de formación su principal canal de entrada a la organización. Retail Action Project (Proyecto de Acción en el Comercio al Público), por ejemplo, ofrece formación especializada en comercio para trabajadores o demandantes de empleo en el sector. La formación ofrecida se orienta hacia el desempeño profesional, pero contiene un fuerte contenido sociopolítico, para conocer los derechos, promover la sindicalización y concienciar a los trabajadores sobre las diferentes formas de opresión y la necesidad de actuar frente a ellas de manera organizada. Denominan a su modelo service to organizing (de servicio a la organización). Igualmente, el Proyecto de Justicia Laboral recluta miembros a través de ofrecer formación gratuita o a bajo coste en OSHA (en español, Administración en Seguridad y Salud Ocupacional). Otros centros de trabajadores ofrecen este tipo de formación, pero también cursos de inglés como segundo idioma y otro tipo de capacitaciones para el empleo en determinados sectores (construcción, niñeras, empleadas de hogar, etc.).

Un número elevado de centros ofrece asesoramiento legal a los trabajadores migrantes y de bajos ingresos. Las principales cuestiones atendidas tienen que ver con el derecho laboral, sobre todo 'robo de salario' (reclamación de cantidades), y con la situación administrativa de las personas migrantes. En ocasiones los centros tienen abogados propios, en otras ocasiones son abogados externos que colaboran con la entidad, a veces de manera altruista, a veces cobrando en función de las cantidades económicas que recibe el trabajador como resultado de su demanda. En relación a las demandas de carácter laboral, algunos centros de trabajadores tratan de compaginar la vía legal con la acción directa. En efecto, es frecuente enviar 'delegaciones' para reclamar cantidades adeudadas a los 
empleadores (que son grupos de organizadores y voluntarios que acuden a entregar carta al empresario en la que se indica las cantidades que deben, y que tratan de iniciar una negociación), además de organizar concentraciones en las puertas de los establecimientos y/o las viviendas de los empleadores. Dado que la legislación estadounidense permite reclamar las nóminas o conceptos retributivos no cobrados con una retroactividad de seis años, los centros de trabajadores son capaces de recuperar importantes sumas económicas como resultado de este tipo de acciones.

Otra actividad que realizan con frecuencia los centros de trabajadores (al menos en el 51,61\% de los casos), es la organización en el trabajo, generalmente a través de elecciones para constituir sindicatos en la empresa y negociar convenios colectivos. La campaña en la empresa Hot \& Crusty del Laundry Workers Center (Centro de Trabajadores de Lavandería), desarrollada entre los años 2012 y 2013, es un buen ejemplo de ello, pues dio lugar a la creación de un sindicato independiente y la negociación de un convenio en una empresa de la restauración.

TABLA 2

ACTIVIDADES REALIZADAS POR LOS CENTROS DE TRABAJADORES EN EL ÁREA METROPOLITANA DE NUEVA YORK

\begin{tabular}{|l|c|c|}
\hline \multicolumn{1}{|c|}{ Actividad } & número & proporción \\
\hline Presión política & 25 & $80,64 \%$ \\
\hline Educación pública y formación & 25 & $80,64 \%$ \\
\hline Servicios legales & 20 & $64,51 \%$ \\
\hline Organización en el trabajo & 16 & $51,61 \%$ \\
\hline Bolsa de trabajo & 8 & $25,80 \%$ \\
\hline Servicios sociales & 8 & $25,80 \%$ \\
\hline Desarrollo de 'líderes' & 7 & $22,58 \%$ \\
\hline Idioma (Inglés como segundo idioma) & 5 & $16,13 \%$ \\
\hline Investigación & 5 & $16,13 \%$ \\
\hline Actividades culturales y comunitarias & 5 & $16,13 \%$ \\
\hline Otras actividades (vivienda, justicia global, justicia racial...) & 3 & $9,67 \%$ \\
\hline
\end{tabular}

Fuente: elaboración propia. 
Además de estas actividades, los centros de trabajadores realizan otras actividades, como: crear bolsas de trabajo (de niñeras, trabajadores de construcción, cooperativas de limpieza...); dar información sobre subsidios a personas en situación de exclusión; formar 'líderes' (que definen como aquellos miembros más implicados en la vida y actividades de la organización); formación en inglés como segundo idioma; investigaciones sobre condiciones laborales; actividades culturales y comunitarias; y otras actividades en áreas como la vivienda, la justicia global y la opresión racial.

En términos generales, puede afirmarse que los centros de trabajadores se diferencian de las organizaciones sindicales, no sólo por su estatus administrativo (como entidad no lucrativa), sino sobre todo por su modelo de organizing, que incluye dirigirse a outsiders del mercado laboral y conectar más la comunidad y el centro de trabajo - por ello se ha les ha ubicado con frecuencia dentro de la categoría de "sindicalismo comunitario" (Fine, 2005; Moody, 2009).

\section{LA FORMACIÓN DE UN CAMPO ORGANIZATIVO}

La existencia de un campo diferenciado se deriva de la tendencia de fuerzas isomórficas, que hacen que las organizaciones tiendan a parecerse entre sí y, por tanto, tiendan a dibujarse ciertas fronteras frente a otro tipo de entidades. Los sistemas de financiación tienen un importante peso. Obtener fondos de fundaciones privadas o subvenciones públicas requiere una especialización en el personal (redactar proyectos, justificar facturas, llevar la contabilidad...). La regulación estatal de este tipo de entidades también induce a los centros de trabajadores a adoptar estructuras similares. Igualmente, hay importantes procesos de imitación entre organizaciones. El mimetismo se realiza de varios modos: el trasvase de personal de unos centros a otros; la divulgación de casos de éxito en campañas, estructuras o iniciativas; los trabajos académicos sobre los centros de trabajadores —en especial, el libro de Fine (2006)—; y las densas redes de colaboración entre centros, que hacen de canales por los que circula información sobre modelos organizativos, estilos de organizing y campañas.

Pero un campo organizativo no es sólo un espacio de cooperación, también es un espacio de competición (Roca, 2006). Los cen- 
tros de trabajadores compiten por recursos escasos y rivalizan con diferentes perspectivas acerca de cuáles son los modelos y repertorios más adecuados para defender a los trabajadores migrantes y de bajos salarios. Mientras algunos centros, como Make the Road o New Immigrant Community Empowerment (NICE), tienen una amplia plantilla y manejan presupuestos anuales de varios millones de dólares, otros centros de trabajadores pequeños critican que los centros mayores copen el espacio, absorban a centros más pequeños y constituyan una amenaza para la supervivencia de los centros de menor tamaño. Precisamente los centros más pequeños son los que con frecuencia ponen más énfasis en la acción directa ante los abusos patronales y son fuente de innovación social, logrando, por ejemplo, alcanzar a nuevos grupos de trabajadores.

Es preciso apuntar que no sólo existen factores que empujan hacia el isomorfismo o la homogeneidad. Como en otros campos organizativos estudiados (Roca, 2014), existen factores que inducen a la heterogeneidad. Algunos trabajos identifican distintos modelos de centros de trabajadores: por ejemplo, en función del grado de influencia de sindicatos y de su priorización de los asuntos laborales sobre los comunitarios (Fine, 2005); o la proporción de actividades de presión política, organizing o provisión de servicios que realizan (Fine, 2006). No obstante, las diferencias también residen en el tipo de alianzas, las ideas políticas o religiosas que inspiran a los dirigentes, o los repertorios de protesta, las estrategias políticas, o las estructuras organizativas. En efecto, hay organizaciones que priorizan el trabajo de presión política sobre los gobiernos municipal y estatal, mientras otras ponen el acento en las cuestiones de lugares de trabajo, o de los barrios. Hay organizaciones vinculadas formalmente a sindicatos y a organizaciones religiosas; otras son independientes, y algunas mantienen vínculos informales con organizaciones políticas. Hay organizaciones en las que el poder interno está distribuido, en otras está más concentrado en manos de figuras carismáticas o la junta directiva. Hay centros de trabajadores en los que el trabajo se encuentra formalmente muy dividido y especializado, mientras en otras la especialización funcional es mucho más flexible.

Por último, los centros de trabajadores se suelen especializar en determinadas industrias o mercados de trabajo, que además suelen estar bien delimitados geográficamente. Así, por ejemplo, el Proyecto de Justicia Laboral trabaja con empleadas de hogar y trabajadores de construcción que ofrecen sus servicios en determinadas 
"paradas" o street corners (espacios públicos en los que los empleadores, generalmente judíos jasídicos, los seleccionan) en el distrito de Brooklyn. El Laundry Workers Center organiza a trabajadores de pequeñas lavanderías (aunque también de restaurantes) repartidos por toda la ciudad. Brandworkers se centra en empresas de industria de comida localizadas en un polígono industrial en Long Island City (Queens), en las que se emplean migrantes, en su mayoría latinoamericanos. Y el Chinesse Staff and Workers Association organiza a trabajadores de restaurantes chinos de Manhattan (principalmente en la zona de Chinatown). El punto en común de todos esos sectores es la elevada precariedad e informalidad laboral, la concentración de mano de obra migrante, constituyendo mercados de trabajo propiamente secundarios.

\section{CONCLUSIONES}

La globalización económica conforma nuevos modelo migratorios caracterizados por un doble proceso: la expulsión de grandes contingentes de trabajadores de sus países de origen y su inserción en mercados de trabajo secundarios, segmentados y altamente informalizados en los países de destino. Esta doble realidad constituye un reto para las organizaciones sindicales tradicionales, que oscilan entre la acogida, mediante el desarrollo de programas de inserción laboral y social de los trabajadores migrantes y el rechazo, en la medida en que los perciben como una amenaza para la regulación de los mercados laborales y para los trabajadores nacionales. En este contexto la respuesta de los trabajadores migrantes se ha concentrado en el desarrollo de formas organizativas propias (englobadas dentro de la categoría de sindicalismo comunitario) que presentan como principal activo el recurso a la redes que se conforman y (re) crean para adaptarse a las nuevas condiciones de trabajo y de vida de estas poblaciones migrantes. Su creatividad proviene del recurso a las redes laborales y residenciales para desarrollar actividades sindicales, parasindicales, de formación laboral y de aprendizaje del inglés, de conocimiento y reinvindicación de sus derechos laborales y políticos y para el desarrollo de actividades comunitarias. El recurso a estas redes constituye además una respuesta espacializada adaptativa a las condiciones de unas economías y sociedades en red 
donde la segmentación y la precarización marcan y condicionan las vidas de los migrantes.

En este sentido, los centros de trabajadores son un modelo innovador y socialmente creativo que constituyen lo que en este artículo hemos conceptuado como campo organizativo. Sin embargo, es precisamente su conformación en red lo que dificulta establecer un modelo isomórfico, ya que los centros, aunque puedan presentar características similares en los ámbitos de su constitución formal y en la adopción de medidas y acciones que han probado su éxito previamente, dependen tambien de manera determinante del tipo de redes que constituyen su base organizativa: su mayor o menor relación con las organizaciones sindicales, agentes comunitarios y agentes políticos, el tipo de liderazgo que los caracteriza, la ideología política o la adscripción religiosa junto a otros factores diferenciales, son elementos que definen sus acciones y estrategias.

Pese a su heterogeneidad, parece observarse una cierta tendencia a la institucionalización en la medida en que algunos centros van consolidando su existencia. Por institucionalización entendemos el establecimiento de relaciones más solidas y consolidadas con los agentes políticos y las entidades del Tercer Sector, una tendencia al crecimiento mediante la incorporación de otros centros de trabajadores, y una apuesta por la profesionalización de las actividades a través la contratación de personal especializado. Otros centros, por el contrario, se caracterizan por priorizar básicamente la acción directa y la reivindicación en los lugares de trabajo, constituyendo una rica fuente de innovación social. Sin embargo, lo que los centros de trabajadores tienen en común es su existencia como actores sociales singularizados y con capacidad de agencia determinante en el ámbito de la inserción social de las poblaciones inmigrantes en los Estados Unidos.

\section{REFERENCIAS}

Avedaño, A., y Hiatt, J. (2012). Worker self-organization in the new economy: The AFL-CIO's experience in movement building with community-labour partnerships. Labour, Capital and Society, 45(1): 66-95.

Black, S. J. (2005). Community Unionism: A Strategy for Organizing in the

New Economy. New Labor Forum, 14(3): 24-32. 
Blake, J. (2012). Professionalism and the third sector. Paper presented at the Annual Meeting of the ISTR $10^{\text {th }}$ International Conference.

BLS (2019). New York City Economic Summary. Update April 03, 2019. Washington DC: Bureau of Labor Statistics. https:/www.bls.gov/regions/ economic\%E2\%80\%90summaries.htm

Bowles, S., y Gintis, H. (1975). The problem with human capital theory-A Marxian critique. American Economic Review, 65(2): 74-82.

Braun, V., \& Clarke, V. (2006) Using thematic analysis in psychology. Qualitative Research in Psychology, 3, 77-101.

Connolly, H., Marino, S., y Martínez Lucio, M. (2014). Trade union renewal and the challenges of representation: Strategies towards migrant and ethnic minority workers in the Netherlands, Spain and the United Kingdom. European Journal of Industrial Relations, 20(1): 5-20.

Connolly, H., Marino, S., y Martínez Luicio, M. (2019). Immigrants and trade unions in the European context. The politics of social inclusion and labor representation. Ithaca and London: ILR Press.

DiMaggio, P. J., y Powell, W. W. (1983). The iron cage revisited: Institutional isomorphism and collective rationality in organizational fields. American Sociological Review, 48: 147-160. doi: 10.2307/2095101

Fielding, T. (2010). Migration in a time of crisis: A simple conceptual framework applied to East Asian migrations (Working Paper $\mathrm{n}^{\circ}$ 63). Brighton: University of Sussex, Sussex Centre for Migration Research.

Fine, J. (2005). Community unions and the revival of the American labor movement. Politics \& Society, 33(1): 153-199. doi: 10.1177/0032329204272553

Fine, J. (2006). Worker centers: organizing communities at the edge of the dream. Ithaca, NY: Economic Policy Institute/Cornell University Press.

Fine. J. (2011). New forms to settle old scores: updating the worker centre story in the United States. Relations Industrielles/Industrial Relations, 66: 604-27.

Fine, J., Narro, V., y Barnes, J. (2019). Understanding worker center trayectories. En J. Fine, L. Burnham, K. Griffith, M. Ji, V. Narro y S. Pitts (Eds.), No one size fits all: Worker organization, policy, and movement in a new economic Age (pp. 7-38). Campaign, IL: LERA.

Gordon, D. M. (1972). Theories of poverty and underemployment. Lexington, MA: DC Heath.

Gordon, D. M., Edwards, R., y Reich, M. (1986). Trabajo segmentado. Trabajadores divididos: La transformación histórica del trabajo en los Estados Unidos. Madrid: Ministerio de Trabajo y Seguridad Social.

Hambrick, D. C., Finkelstein, S., Cho, T. S., y Jackson, E. M. (2005). Isomorphism in reverse: Institutional theory as an explanation for recent increases in intra-industry heterogeneity and managerial discretion. Research in Organizational Behavior, 26: 307-350. doi: 10.1016/S01913085(04)26008-7 
Heery, E., Williams, S., y Abbott, B. (2012). Civil society organizations and trade unions: cooperation, conflict, indifference. Work, Employment and Society, 26(1): 145-160. doi: 10.1177/0950017011426302

Hopkins, T., y Wallerstein, I. (1977). Patterns of development of the modern World-system. Review, 1(2): 11-145.

Jarayaman, S., y Ness, I. (2005). Introduction. En S. Jarayaman y I. Ness (Eds.), The new urban immigrant workforce. Innovative models for labor organizing (pp. 3-8). New York, London: M E Sharp.

Jenkins, S. (2002). Organizing, advocacy, and member power: A critical reflection. Working USA, 6(2):56-89

King, R., y Black, R. (1997). Southern Europe and the new immigrations. Brighton: Sussex Academic Press.

Leiter, J. (2005). Structural isomorphism in Australian non-profit organizations. Voluntas, 16(1): 1-31. doi: 10.1007/s11266-005-3230-1

Lesser, G., y Batalova, J. (2017, abril 17). Inmigrantes centroamericanos en Estados Unidos. Migration Information Source. Recuperado de https:// www.migrationpolicy.org/article/inmigrantes-centroamericanos-en-losestados-unidos

Lopez, S. H. (2004). Reorganizing the Rust Belt: An inside study of the American labor movement. Berkeley: University of California Press.

Manheim, J. B. (2017). The emerging role of worker centers in union organizing. An update and a supplement. Washington, DC: US Chamber of Commerce.

Marino, S., Pennix, R., y Roosblad, J. (2015). Trade unions, immigration and immigrants in Europe revisited: Unions' attitudes and actions under new conditions. Comparative Migration Studies, 3:1. doi: 10.1007/s40878015-0003-x

Martín Díaz, E., Melis Maynar, A., y Sanz Casas, G. (2004). Mercados de trabajo e inmigración extracomunitaria en la agricultura mediterránea. Sevilla: Junta de Andalucía, Consejería de Asuntos Sociales.

Martínez Lucio, M. (2017). Organising without knowing it? The curious case of para-organising-style campaigns in southern Europe and the case of trade union elections in Spain. Transfer 23(1): 89-94.

Massey, D. S., y Pren, K. A. (2013). The United States war against immigration. Paradoxical effects. Doc Anal Geogr, 59(2): 209-237.

Mayring, P. (2007). On generalization in qualitatively oriented research [23 paragraphs]. Forum Qualitative Sozialforschung / Forum: Qualitative Social Research, 8(3): Art. 26, http://nbn-resolving.de/urn:nbn:de:0114fqs0703262

McBride, J., y Greenwood, I (eds) (2009). Community Unionism: A Comparative Analysis of Concepts and Contexts. Basingstoke: Palgrave Macmillan.

Milkman, R. (2000). L.A. Story. Immigrant workers and the future of the US Labor Movement. New York: Russell Sage Foundation. 
Milkman, R. (2013). Back to the Future? US Labour in the New Gilded Age. British Journal of Industrial Relations, 51(4): 645-665.

Milkman, R. (2014). Introduction: Toward a new labor movement? Organizing New York City's precariat. En R. Milkman y E. Ott (Eds.), New Labor in New York. Precarious Workers and the Future of the Labor Movement (pp. 1-23). Ithaca and London: Cornell University Press.

MOIA. (2018). State of our immigrant city. Annual Report March 2018. Nueva York: NYC Mayor's Office for Immigrant Affairs. https://www1.nyc.gov/ assets/immigrants/downloads/pdf/moia_annual_report_2018_final.pdf

Moody, K. (2009). Immigrant workers and labour/community organisations in the United States. En J. McBride y I. Greenwood (Eds.), Community unionism. A comparative analysis of concepts and contexts (pp. 139-159). Basingstoke: Palgrave MacMillan.

Mora, C. (2014). Making Hispanics. How activists, bureaucrats and media constructed a new American. Chicago: University of Chicago Press.

Mottura, G. (1992, septiembre). Forme della presenza extracomunitaria nella agricoltura italiana: resultati di una prima esplorazione. Ponencia presentada al curso de la UIMP Trabajadores inmigrantes en la agricultura mediterránea, Valencia, España.

Murray, G. (2017). Union renewal: what can we learn from three decades of research? Transfer: European Review of Labour and Research, 23(1): 9-29. doi: $10.1177 / 1024258916681723$

Ness, I. (2005). Immigrants, unions, and the new U. S. labor market. Philadelphia: Temple University Pres.

Pennix, R., y Roosblad, J. (Eds). (2001). Trade unions, immigration and immigrants in Europe, 1960-1993: A comparative study of the actions of trade unions in seven West European countries. London: Berghahn Books.

Piore, M. J. (1969.) Impact of the labor market upon the design and the selection of production techniques within the manufacturing plan. Quarterly Journal of Economics, 82(4): 602-620.

Piore, M. J. (1979). Birds of Passage: Migrant Labour and Industrial Society. Cambridge: Cambridge University Press.

Pugliese, G. (1992, septiembre). L'immigrazione in Italia: un confronto tra Nord e Sur. Ponencia presentada al curso de la UIMP Trabajadores inmigrantes en la agricultura mediterránea, Valencia, España.

Roca, B. (2006). Entre la competencia y la cooperación: la construcción de redes entre las Organizaciones No Gubernamentales de Desarrollo en Andalucía. Redes. Revista hispana para el análisis de redes sociales, 11(2). https://revistes.uab.cat/redes/article/download/v11-n2-roca/94-pdf-es

Roca, B. (2014). Conflicto y cambio en el tercer sector. Carisma, control externo y heterogeneidad en las ONGD. Papers: Revista de Sociología, 99(1): 97-118.

Roca, B. (2016). Transformaciones en el trabajo y movimiento sindical. Propuestas para una renovación necesaria. Madrid: Fundación Alternativas. 
Roca, B. (2019). Analyzing Isomorphism in Nongovernmental Organizations: Ethical Dilemmas in Ethnographic Fieldwork. SAGE Research Methods Cases, 2: 1-10

Sassen, S. (2005). The Global City: Introducing a concept. Brown Journal of World Affairs, 11: 27-43.

Sassen, S. (2014). Expulsions: Brutality and complexity in the global economy. Cambridge, MA: The Belknap Press of Harvard University Press.

Semuels, A. (2018, junio 27). Is This the End of Public-Sector Unions in America? The Atlantic. Recuperado de https://www.theatlantic.com/ politics/archive/2018/06/janus-afscme-public-sector-unions/563879/

Silverman, D. (2009). Doing Qualitative Research. London: SAGE.

Sullivan, R. (2010). Revitalization and the role of community-based organizations organizing workers in the space between unions: Unioncentric labor. Critical Sociology, 36(6): 793-819.

Wills, J., y Simms, M. (2004). Building reciprocal community unionism in the UK. Capital and Class, 28(1): 59-84. 